\title{
Failure of propranolol to improve exercise tolerance in patients with mitral stenosis in sinus rhythm
}

\author{
MAYER M BASSAN, * JOSEPH MICHAELI, ODED SHALEV $\dagger$ \\ From the $\nmid$ Department of Internal Medicine, Hadassah Hebrew University Medical Center, Mt Scopus; and \\ the ${ }^{\star}$ Jerusalem Heart Clinic of Kupat Holim, Jerusalem, Israel
}

SUMMARY Propranolol reduces left atrial pressure at rest and during exercise in patients with mitral stenosis by lowering cardiac output and heart rate. Ten patients (aged 19-56) with moderate to severe isolated mitral stenosis were studied to determine whether propranolol increased their exercise tolerance. All were in sinus rhythm and free of left or right ventricular failure. Patients were trained in an individually graded bicycle or treadmill exercise protocol that provoked a reproducible degree of near maximal dyspnoea during the second three minute stage of exercise. Propranolol ( $80 \mathrm{mg}$ or $120 \mathrm{mg}$ ) or matching placebo in two or three divided daily doses was given for one week in random double blind fashion. Exercise testing and questioning about subjective clinical response were carried out at the end of each week by an investigator who was unaware of the patient's heart rate. During propranolol treatment the heart rate was 19 beats/minute slower at rest and 38 beats/minute slower at peak exercise, but there was no change in mean exercise time to dyspnoea (274 s during propranolol vs $283 \mathrm{~s}$ during placebo). Four patients felt worse during the propranolol week, one patient felt better during the propranolol week, and five patients felt no difference between the two weeks.

Propranolol did not improve objective or subjective exercise tolerance in patients with isolated mitral stenosis in sinus rhythm.

The potential beneficial effect of $\beta$ blockade in patients with mitral stenosis has been studied over the past 20 years. $^{1-3}$ The rationale for these investigations was the understanding that the pressure gradient across a stenotic mitral valve rises exponentially as the heart rate and cardiac output increase. ${ }^{4}$ Thus the increases in heart rate and cardiac output that occur during exercise lead to higher left atrial and pulmonary venous pressure, which are the presumed cause of the exertional dyspnoea of mitral stenosis. ${ }^{5} \beta$ blockade, by blunting the rises in heart rate and cardiac output during exercise, and thereby increasing the diastolic filling period and reducing transvalvar flow, would be expected to reduce the increase in pulmonary venous pressure at any given level of exercise. Indeed, in studies in which intravenous pronethalol ${ }^{1}$ or propranolol ${ }^{23}$ were given to patients with mitral stenosis during diagnostic car-

Requests for reprints to Dr Mayer M Bassan, Jerusalem Heart Clinic, 12 Paran Street, Jerusalem, Israel.

Accepted for publication 21 April 1987 diac catheterisation, heart rate and cardiac output were reduced, with an accompanying fall in left atrial pressure at rest and a reduced rise during exercise. $^{1-3}$ The usual conclusion of these studies was that long term treatment with $\beta$ blockers would be likely to benefit patients with mitral stenosis.

Cumming and Carr, however, suggested that the negative inotropic effect of propranolol on the left ventricle, as well as the reduction in cardiac output, would be likely to cause an overall deterioration in cardiac performance, and thus the drug would not be of value in mitral stenosis. ${ }^{6}$ Furthermore, $\beta$ blockade has not gained widespread acceptance in the treatment of patients with mitral stenosis. ${ }^{7}$ The only investigation of the effect of $\beta$ blockade on exercise testing found that the treatment was of benefit; but the study was flawed by a single blind protocol and a poorly defined end point for cessation of exercise. ${ }^{8}$

To investigate further the potential benefit of the treatment of mitral stenosis with $\beta$ blockade we have used an individually formulated exercise protocol to evaluate in a double blind trial the effect of propranolol on exercise tolerance in patients with mitral stenosis. 


\section{Patients and methods}

We studied ten patients with isolated mitral stenosis. The diagnosis was established by typical auscultatory, electrocardiographic, radiographic, and echocardiographic findings. The patients were in New York Heart Association functional class II or III and five had valve surgery 1-24 months after completing the study protocol. In eight patients the mitral valve area was estimated by cross sectional echocardiography while in the other two an estimation was available in the report of the operation (table). No patient had echocardiographic evidence of left ventricular enlargement or abnormal left ventricular function. None of the patients had evidence of right heart failure. No patient had obstructive lung disease or any other contraindication to $\beta$ blockade.

Patients were trained according to an individual exercise protocol to reach a reproducible degree of near maximal dyspnoea. Five patients performed bicycle exercise and five exercised on a treadmill. Increments per three minute stage were $20 \mathrm{~W}$ and 1.0 $\mathrm{kph}$ respectively. In the training phase the patient became accustomed to the exercise laboratory and staff, recognised his dyspnoea end point, and attained a certain amount of physical conditioning. Achievement of stable performance (less than a 30 second variation in exercise duration) usually required 8-12 exercise bouts over several weeks with $2-3$ bouts per session. There was invariably an improvement of at least one three minute stage during the training phase, and the starting level in watts or incline/speed for the study phase was chosen so that end point dyspnoea was reached after 3-6 minutes of exercise. At the final training session the patient was given a test dose of $40 \mathrm{mg}$ of propranolol and was observed for several hours for possible adverse effects as well as for the degree of induced bradycardia.

During the study phase propranolol or matching placebo were given for one week each in randomised, double blind crossover fashion. The daily dose of propranolol was 80 or $120 \mathrm{mg}$ (in two or three divided doses), according to body weight and the degree of bradycardia induced by the test dose. During a third week all patients except case 1 were given half the previous propranolol dose single blind. At the end of each week the patient was questioned in detail about beneficial or adverse effects by an investigator who did not know the patient's heart rate.

An exercise evaluation consisting of two bouts one hour apart was carried out at the end of each week. In order to maintain the double blind character of the study two investigators were present and one of them remained unaware of the patient's heart rate. Patients exercised according to the protocol that was established during the training phase. Patients were fasting. The dose of study medication was taken one and a half to two hours before the test. The patient's electrocardiogram was monitored continuously, and heart rate was recorded at rest, at three minutes, and at peak exercise. The investigator who remained unaware of the patient's heart rate stood out of view of the monitor, the recorder, and the clock, and decided with the patient when the end point of near maximal dyspnoea had been reached. This investigator then signalled the other investigator to stop the timer and record the heart rate. The blinded investigator then counted the number of respirations during the first minute after the end of exercise.

In patients 5-10 the blinded investigator measured the peak expiratory flow at the end of each week with a Wright peak flow minimeter.

We explained to each patient that this was a research study and obtained their informed consent. The study was approved by the institutional committee on research on human subjects.

\section{STATISTICAL ANALYSIS}

We used a mean of the two values for each exercise variable from the two bouts to represent the patient's exercise performance for that day. We used Student's $t$ test for matched pairs to compare heart rates

Table Data on individual patients

\begin{tabular}{|c|c|c|c|c|}
\hline Patient & Age & Sex & Mitral valve area & Starting exercise level \\
\hline $\begin{array}{l}1 \\
2 \\
3\end{array}$ & $\begin{array}{l}56 \\
41 \\
43\end{array}$ & $\begin{array}{l}\mathbf{F} \\
\mathbf{F} \\
\mathbf{M}\end{array}$ & $\begin{array}{l}1 \cdot 1 \mathrm{~cm}^{2} \mathrm{E} \\
\text { "Severe stenosis" S } \\
0.7 \mathrm{~cm}^{2} \mathrm{E} \\
\text { "Severe stenosis" S }\end{array}$ & $\begin{array}{l}40 W \\
40 W \\
60 W\end{array}$ \\
\hline $\begin{array}{r}4 \\
5 \\
6 \\
7 \\
8 \\
9 \\
10\end{array}$ & $\begin{array}{l}39 \\
27 \\
35 \\
40 \\
49 \\
19 \\
38\end{array}$ & $\begin{array}{l}\mathbf{F} \\
\mathbf{F} \\
\mathbf{F} \\
\mathbf{M} \\
\mathbf{F} \\
\mathbf{M}\end{array}$ & $\begin{array}{l}1.0 \mathrm{~cm}^{2} \mathrm{~S} \\
1.4 \mathrm{~cm}^{2} \mathrm{E} ; 1.0 \mathrm{~cm}^{2} \mathrm{~S} \\
1.7 \mathrm{~cm}^{2} \mathrm{E} \\
1.3 \mathrm{~cm}^{2} \mathrm{E} \\
0.9 \mathrm{~cm}^{2} \mathrm{E} \\
1.2 \mathrm{~cm}^{2} \mathrm{E} ; 1.1 \mathrm{~cm}^{2} \mathrm{~S} \\
0.8 \mathrm{~cm}^{2} \mathrm{E}\end{array}$ & $\begin{array}{l}10 \mathrm{~W} \\
40 \mathrm{~W} \\
14 \%, 5 \cdot 0 \mathrm{kph} \\
14 \%, 4 \cdot 0 \mathrm{kph} \\
14 \%, 4.0 \mathrm{kph} \\
10 \%, 3 \cdot 0 \mathrm{kph} \\
16 \%, 3.5 \mathrm{kph}\end{array}$ \\
\hline
\end{tabular}

E, Echocardiographic estimate; S, estimate at operation. 
and exercise times during the placebo, propranolol, and half-dose propranolol weeks. Results are expressed as means (1 SE).

\section{Results}

Six patients received propranolol during the second week of the study. Mean heart rates were markedly lower during propranolol treatment than during placebo treatment: 55 (1) beats/min vs 74 (2) beats/min at rest $(p<0.0001) ; 103$ (3) vs 132 (4) beats $/ \mathrm{min}$ at 3 minutes exercise $(\mathrm{p}<0.0001)$; and 115 (5) vs 151 (6) beats/min at peak exercise $(\mathrm{p}<0.0001)$ (fig 1). All exercise bouts were stopped because of dyspnoea, and there was no difference in mean exercise time to near maximal dyspnoea between the propranolol and placebo weeks: 274 (25) s vs 283 (26) s (NS) (fig 2). During the third week, when patients received half the previous propranolol dose, heart rates were $63(2)$ at rest, $115(5)$ at three minutes, and 127 (4) at peak exercise $(p<0.005)$ for all values compared with placebo); but again there was no change in the mean duration of exercise (267 (23) s). The mean number of respirations during the first minute after exercise was 28 during propranolol treatment and 29 during placebo, indicating a similar degree of dyspnoea.

In response to questions about exertional dyspnoea, palpitation, fatigue, and overall wellbeing

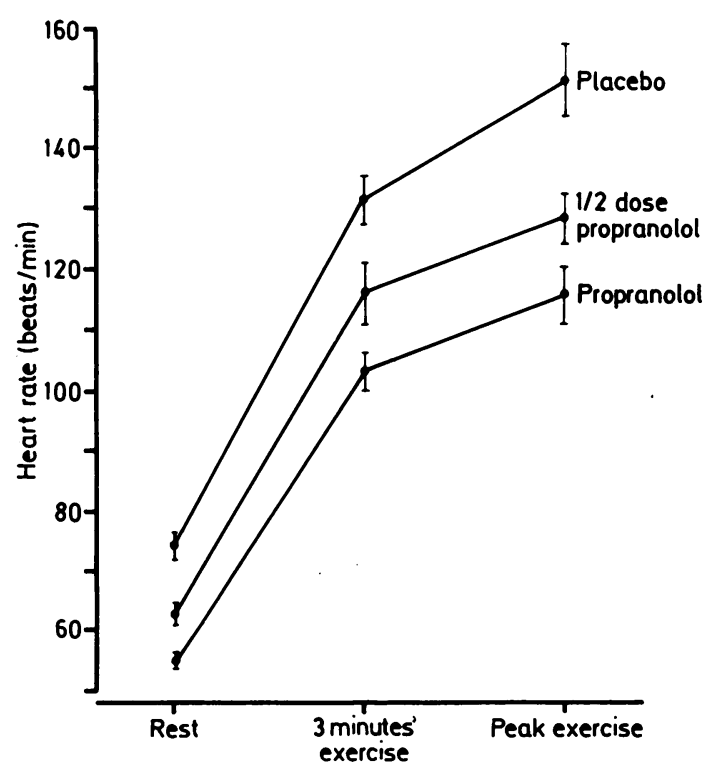

Fig 1 Mean heart rates at rest after three minutes of exercise, and just before stopping exercise because of dyspnoea. Exercise tests were performed at the end of each treatment week.

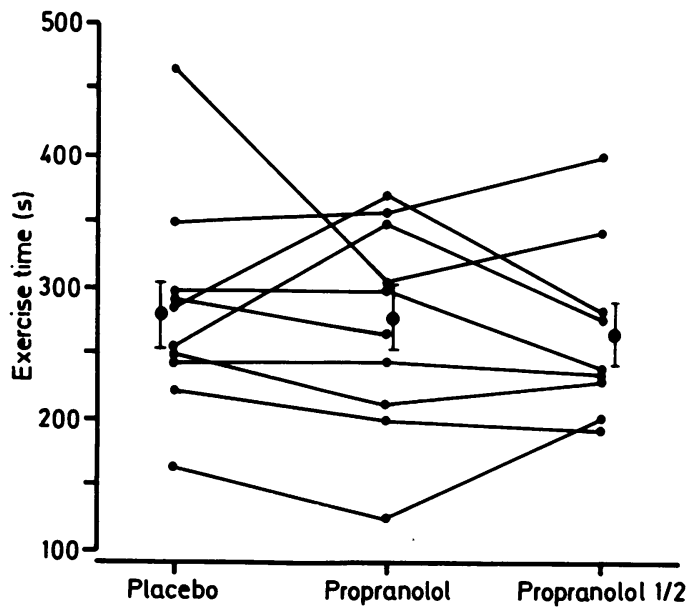

Fig 2 Exercise times to near maximal dyspnoea during the exercise tests performed at the end of each treatment week. Individual results and group means (SE) are shown.

during the two blinded treatment weeks, five patients felt no difference between the two weeks, four patients felt worse on propranolol, and one patient felt slightly better on propranolol.

Among the six patients in whom peak flow rate was measured the mean rates were identical during placebo and propranolol treatment.

\section{Discussion}

Despite haemodynamic observations that suggested that propranolol would be likely to increase exercise tolerance in patients with mitral stenosis, ${ }^{1-3}$ our results indicate that treatment with this drug improves neither subjective nor objective exercise ability. The often poor correlation between improvement in haemodynamic variables in chronic heart failure and improvement in symptoms has been increasingly appreciated in recent years, and formal exercise testing is thus being used more frequently to evaluate changes in functional state in patients in heart failure. ${ }^{9}$ In such cases it is crucial that the exercise test procedure objectively measures exercise capacity, and attention has been called to the subjectivity of symptomatic end points that are influenced by patient motivation and observer bias. ${ }^{9}$ For this reason we trained our patients carefully before the experimental phase until a reproducible end point of near maximal dyspnoea was achieved; after this the study itself was carried out in double blind fashion.

In addition, in the minute immediately after exercise we measured the respiratory rate as a further indication of the degree of dyspnoea achieved. We used an individually formulated exercise protocol 
designed to produce a baseline exercise duration of 3-6 minutes on the model of the Redwood protocol ${ }^{10}$ used in studying angina pectoris. Although exercise duration may be somewhat shorter than with standard exercise protocols, the briefer, more intense exercise is probably more similar to the type of effort that limits patients in their daily activities (for example two flights of stairs or 100 metres uphill). We have considerable experience with such a protocol in evaluating antianginal drugs, and have found that despite the shortness of the exercise, even extensively trained patients are capable of considerable increases in exercise duration after receiving an effective drug. ${ }^{11}$ The end point of "near maximal dyspnoea" was defined to the patient as that degree of breathlessness which would cause him to rest during his ordinary exertion at a time when he was not in a particular hurry. Our subjects each performed a total of approximately 15 exercise bouts during the training and experimental phases of the study and we felt that it would be unfair to require them to exercise repeatedly to maximum dyspnoea or exhaustion. This might have made them less willing to complete the study.

In the only other investigation that used formal exercise testing to study the effect on exercise (it found $\beta$ blockade to be of benefit) the patients were not trained, the end point was defined as "exhaustion, dizziness or severe dyspnoea", and the investigator supervising the exercise tests knew the patient's heart rate and thus could guess whether the patient was receiving a $\beta$ blocker or a placebo. ${ }^{8}$ Our own blinding procedure, however, prevented only the influence of a bias in favour of propranolol. Since the blinded investigator was aware of the patient's baseline exercise performance, a bias against the effectiveness of propranolol could have influenced the decision as to when to terminate an exercise bout. The effect of this potential influence was limited because the blinded investigator could not see the clock. In fact the original hypothesis was that propranolol would be effective; it was only when we were midway through the study that we adopted the opposite view.

Although we did not use large doses of propranolol we added a third week at half dose to be certain that we would not miss a therapeutic effect because of excessive $\beta$ blockade. The reductions in heart rate at rest and at peak exercise were less than on the full doses of propranolol, but there was still no difference in exercise duration.

Although we did not measure pulmonary venous pressure, it is reasonable to assume, given the reductions in resting and exercise heart rates during treatment with $\beta$ blocker, that the usual rise in pulmonary venous pressure seen during exercise in patients with mitral stenosis was blunted. Why then was there no concomitant improvement in exercise tolerance?

Although the classic view has been that the exertional dyspnoea of mitral stenosis is the result of the increased pulmonary venous pressure and volume that occurs during exercise, ${ }^{12}$ the difficulty of separating the effects of pulmonary venous hypertension from the insufficient increase in cardiac output and oxygen delivery to exercising muscles has been long noted. ${ }^{13}$ Failure to find a correlation between the degree of lung rigidity and the degree of dyspnoea suggested that the pulmonary changes in mitral stenosis might not be the primary cause of the dyspnoea.$^{13}$ Recent studies on the ventilatory responses to exercise in patients with chronic left ventricular failure have suggested that the exertional dyspnoea is primarily the result of the insufficient cardiac output and inadequate oxygen transport to working muscle, rather than of the increase in pulmonary venous pressure. ${ }^{1415}$ Although the patients in these studies had left ventricular failure rather than mitral stenosis, ${ }^{1415}$ the presence of the combination of a rise in pulmonary venous pressure in the face of an insufficient increase in cardiac output during exercise in both stages suggests that the same mechanism of dyspnoea may be operating. Thus while $\beta$ blockade in patients with mitral stenosis might have a beneficial effect on pulmonary venous hypertension, the deleterious effect on the possibly more important factor of cardiac output may explain our failure to find a clinical improvement.

An additional explanation for the ineffectiveness of $\beta$ blockade in mitral stenosis may be that right ventricular function is depressed. An abnormal right ventricular response to exercise has been demonstrated in patients with mitral stenosis in the absence of resting right ventricular failure, ${ }^{16}$ and at the same time it has been shown that right ventricular ejection fraction is highly correlated with exercise capacity in patients with chronic left ventricular failure. ${ }^{17}$

Whatever the mechanism, the results of our study, which is the first double blind investigation of this issue, suggest that $\beta$ blockade does not have a role in the treatment of patients with isolated mitral stenosis in sinus rhythm.

This study was supported by a grant from the Mac Ramer Heart Research Scholarship Fund, Flushing, New York.

\section{References}

1 Howitt G, Tinker J, Wade EG. The effect of pronethalol in mitral stenosis. Clin Sci 1965;28:417-26.

2 Meister SG, Engel TR, Feitosa GS, Helfant RH, Franke WS. Propranolol in mitral stenosis during sinus rhythm. Am Heart $J$ 1977;94:685-8. 
3 Giuffrida G, Bonzani G, Betocchi S, et al. Hemodynamic response to exercise after propranolol in patients with mitral stenosis. $A m J$ Cardiol 1979;44:1076-82.

4 Gorlin R, Lewis BM, Haynes FW, Spigel RJ, Dexter L. Factors regulating pulmonary capillary pressure in mitral stenosis. IV. Am Heart $J$ 1951;41:834-54.

5 Braunwald E. Valvular heart disease. In: Braunwald E, ed. Heart disease. A textbook of cardiovascular medicine. Philadelphia: WB Saunders, 1984:1066.

6 Cumming GR, Carr W. Hemodynamic response to exercise after beta adrenergic blockade with propranolol in patients with mitral valve obstruction. Can Med Assoc J 1966;95:527-31.

7 Braunwald E. Valvular heart disease. In: Braunwald E, ed. Heart disease. A textbook of cardiovascular medicine. Philadelphia: WB Saunders, 1984:1072.

8 Klein HO, Sareli P, Schamroth CL, Carim Y, Epstein M, Marcus B. Effects of atenolol on exercise capacity in patients with mitral stenosis with sinus rhythm. Am J Cardiol 1985;56:598-601.

9 Franciosa JA. Exercise testing in chronic congestive heart failure. Am J Cardiol 1984;53:1447-50.

10 Redwood DR, Rosing DR, Goldstein RE, Beiser GD, Epstein SE. Importance of the design of an exercise protocol in the evaluation of patients with angina pectoris. Circulation 1971;43:618-28.
11 Bassan MM, Weiler-Ravell D, Shalev O. Comparison of the antianginal effectiveness of nifedipine, verapamil, and isosorbide dinitrate in patients receiving propranolol: a double-blind study. Circulation 1983;68:567-75.

12 Gazetopoulos N, Davies H, Oliver C, Deuchar D. Ventilation and haemodynamics in heart disease. $\mathrm{Br}$ Heart $J$ 1966;28:1-15.

13 Hayward GW, Knott JMS. The effect of exercise on lung distensibility and respiratory work in mitral stenosis. Br Heart J 1955;17:303-11.

14 Franciosa JA, Leddy CL, Wilen M, Schwartz D. Relation between hemodynamic and ventilatory responses in determining exercise capacity in severe congestive heart failure. Am $J$ Cardiol 1984; 53:127-34.

15 Wilson JR, Ferraro N. Exercise intolerance in patients with chronic left heart failure: relation to oxygen transport and ventilatory abnormalities. $\mathrm{Am} \mathrm{J} \mathrm{Car-}$ diol 1983;51:1358-63.

16 Cohen M, Horowitz SF, Machac J, Mindich BP, Fuster $\mathrm{V}$. Response of the right ventricle to exercise in isolated mitral stenosis. Am J Cardiol 1985;55:1054-8.

17 Baker BJ, Wilen MM, Boyd CM, Dinh H, Franciosa JA. Relation of right ventricular ejection fraction to exercise capacity in chronic left ventricular failure. Am J Cardiol 1984;54:596-9. 